\title{
Fluorometric detection of protein-ligand engagement: The case of phosphodiesterase5
}

\author{
Giulia Di Rocco ${ }^{\mathrm{a}, *}$, Ilaria Martinelli ${ }^{a}$, Salvatore Pacifico ${ }^{\mathrm{b}}$, Remo Guerrini ${ }^{\mathrm{b}}$, Elena Cichero $^{\mathrm{c}}$, \\ Paola Fossa $^{c}$, Silvia Franchini ${ }^{a}$, Silvia Cardarelli ${ }^{d}$, Mauro Giorgi ${ }^{d}$, Marco Sola $^{\text {a }}$, \\ Glauco Ponterini ${ }^{\mathrm{a}, *}$ \\ a Department of Life Sciences, University of Modena and Reggio Emilia, Via Campi 103, 41125 Modena, Italy \\ b Department of Chemical and Pharmaceutical Sciences, University of Ferrara Via Fossato di Mortara 17-19, Ferrara, 44100, Italy \\ ${ }^{\mathrm{c}}$ Department of Pharmacy, Section of Medicinal Chemistry, School of Medical and Pharmaceutical Sciences, University of Genova, Viale Benedetto XV, 3 , \\ 16132 Genova, Italy \\ d Department of Biology and Biotechnology “Charles Darwin”, Sapienza University of Rome, P.le A. Moro 5, 00185 Roma, Italy
}

\section{A R T I C L E I N F O}

\section{Article history:}

Received 3 August 2017

Received in revised form

22 September 2017

Accepted 1 November 2017

\section{Keywords:}

Phosphodiesterase 5

Fluorometric ligand binding detection

Competitive displacement analysis

cGMPS-rhodamine

Förster resonance energy transfer (FRET)

Affinity constant $\left(K_{d}\right)$

\begin{abstract}
A B S T R A C T
Phosphodiesterases (PDEs) regulate the intracellular levels of cAMP and cGMP. The great clinical success of the PDE5 inhibitors, Sildenafil (Viagra), Vardenafil (Levitra) and Tadalafil (Cialis) has led to an increasing interest for this class of enzymes. Recent studies have shown a correlation between tumor growth and PDE5 overexpression, making PDE5-selective inhibitors promising candidates for cancer treatment. The search for such inhibitors rests today on radioactive assays. In this work, we exploit the conserved catalytic domain of the enzyme and propose a faster and safer method for detecting the binding of ligands and evaluate their affinities. The new approach takes advantage of Förster Resonance Energy Transfer (FRET) between, as the donor, a fluorescein-like diarsenical probe able to covalently bind a tetracysteine motif fused to the recombinant PDE5 catalytic domain and, as the acceptor, a rhodamine probe covalently bound to the pseudosubstrate cGMPS. The FRET efficiency decreases when a competitive ligand binds the PDE5 catalytic site and displaces the cGMPS-rhodamine conjugate. We have structurally investigated the PDE5/cGMPS-rhodamine complex by molecular modelling and have used the FRET signal to quantitatively characterize its binding equilibrium. Competitive displacement experiments were carried out with tadalafil and cGMPS. An adaptation of the competitive-displacement equilibrium model yielded the affinities for PDE5 of the incoming ligands, nano- and micromolar, respectively.
\end{abstract}

(c) 2017 Elsevier B.V. All rights reserved.

\section{Introduction}

Phosphodiesterase enzymes (PDEs) are phosphohydrolases that selectively hydrolyse ubiquitous second messengers (cGMP and cAMP) thereby regulating their signaling pathways and downstream biological effects [1-3]. PDEs accomplish different biological roles, including smooth muscle relaxation and blood pressure

Abbreviations: PDE5, Phosphodiesterase 5; PDE5C, Phosphodiesterase 5 catalytic domain; PDE5C-TC, phosphodiesterase 5 catalytic domain modified with a tetracysteine tag; PDE5C-TC-FlAsH, complex obtained by conjugation of the FlAsH fluorophore to Phosphodiesterase 5 Catalytic domain modified with a tetracystein tag; FlAsH-EDT $2,4^{\prime} 5^{\prime}$-Bis(1,3,2-dithiarsolan-2-yl)-fluorescein; cGMPS, $2^{\prime}$-(6-Aminohexylcarbamoyl)guanosine-3',5'-cyclic monophosphorothioate.

* Corresponding authors.

E-mail addresses: giulia.dirocco@unimore.it (G. Di Rocco), glauco.ponterini@unimore.it (G. Ponterini). regulation, platelet aggregation and disaggregation, ion channel conductance and neurotransmission, cell growth, apoptosis and cellular mobility and contractility [2]. PDEs have been exploited pharmacologically for more than half a century and some of the most successful drugs worldwide like Sildenafil (Viagra), Vardenafil (Levitra) and Cialis (tadalafil) affect PDE5 function. Therefore, these drugs are subjected to extensive mechanistic investigations and clinical trials [6]. PDEs comprise a 21-gene super family categorized into 11 families. Among them, type 5 phosphodiesterase (PDE5), of which three isoforms (PDE5A1, PDE5A2 and PDE5A3) are known, is responsible for CGMP hydrolysis [1-3]. PDE proteins, with a few exceptions, contain a highly conserved catalytic domain, but differ in the regulatory domain at the $\mathrm{N}$-terminus [3]. Recently, functional variants of PDE genes have been suggested to play a role in predisposition to tumors [1,4-7]. These activities would strongly benefit from the development of fast, sensitive and reliable tools for rapidly screening potential PDE5 inhibitors. Herein, 
we demonstrate that the tetracysteine-tag technology applied to the PDE5 catalytic domain (PDE5C, coding region Glu536-Gln860) is a promising approach in this respect. This method exploits the binding of a fluorescent probe, the fluorescein diarsenical hairpin binder (FlAsH), to a short peptide sequence which includes four cysteine residues, namely Cys-Cys-Xaa-Yaa-Cys-Cys (CCXYCC, in which $\mathrm{X}$ and $\mathrm{Y}$ denote any amino acid) to be engineered on the target protein $[8,9]$. Site-selective labelling of proteins in living cells can be achieved with this method that has been applied to address a variety of issues involving detection of proteinprotein interactions [10-12]. This molecular technology has also been recently employed to detect and quantitatively characterize the engagement of a small-molecule inhibitor with its target enzyme in a cell lysate [13] but, to our knowledge, it has never be applied to purified enzyme. We have expressed the catalytic domain of the PDE5A2 (identifier: 076074-2) with a genetically encoded 6xHis tag at the N-terminus and the 6-amino-acid motif CCPGCC (TC) at the $C$ terminus. This tetra-cysteine motif proved able to covalently bind the biarsenical fluorescein probe (FlAsHFig. 1) $[9,11]$. The complex between the fluorescent protein and the non hydrolysable pseudosubstrate cGMPS, tagged with rhodamine, was obtained and used for displacement studies by the known PDE5 inhibitor tadalafil and by cGMPS. A Förster resonance energy transfer (FRET)-based determination produced the binding constant for the fluorescent cGMPS-rhodamine pseudosubstrate. Similarly, in experiments where either tadalafil or cGMPS were added to PDE5C-TC-FlAsH/cGMPS-rhodamine complex solutions, the observed decrease in the efficiency of FRET between FlAsH and rhodamine enabled us to prove the occurrence of ligand displacement as well as estimate the affinity constant of the two ligands for the PDE5 catalytic site. In addition, we derived structural information on the binding mode of the pseudosubstrate by molecular docking calculations of the cGMPS-rhodamine at the modelled PDE5A2 catalytic site (PDE5C). Because the presently available X-ray diffraction structures for PDEs are limited to the catalytic domains, the homology model of the related human PDE5A2 isoform created by us was limited to this portion of the protein.

\section{Materials and methods}

\subsection{Materials}

Molecular Biology Reagents: Primers for the cloning of the PDE5A2 catalytic domain were purchased from Eurofins Genomics S.r.l (Italy). The plasmid pLATE 52, IPTG ( $\beta$-Dthiogalactopyranoside), $\beta$-mercaptoethanol and phenylmethylsulfonyl fluoride (PMSF) were all purchased from Thermo Fisher Scientific (Waltham, MA, USA). Tris(2-carboxyethyl)phosphine hydrochloride (TCEP) was purchased from Sigma-Aldrich (St. Louis, MO, USA)

Ammonium bicarbonate (AMBIC), dithiothreitol (DTT), iodoacetamide (IAA) were all purchased by Sigma-Aldrich. FlAsH $-\mathrm{EDT}_{2}$ was from Santa Cruz Biotechnology (Dallas, TX, USA).

2'-(6-Aminohexylcarbamoyl)guanosine-3',5'-cyclic monophosphorothioate (RP-2'-AHC-cGMP) and the sulforhodamine B-X succinimidyl ester (Rhodamine Red- $\mathrm{X}^{\mathrm{TM}}$ ) were purchased from BioLog (Bremen, Germany) and Thermo Fisher Scientific, respectively. Tadalafil was purchased from Sigma-Aldrich.

The solvents Trifluoroacetic acid (TFA), Acetonitrile $\left(\mathrm{CH}_{3} \mathrm{CN}\right)$ and N,N-Diisopropylethylamine (DIPEA), were purchased from SigmaAldrich.

\subsection{Production of recombinant PDE5C-TC}

The wild type PDE5 catalytic domain (PDE5C), Glu536-Gln860 coding region, was constructed from the full length human PDE5A2 cDNA (kindly provided by Federica Barbagallo of the "La Sapienza" University, Rome) as the template. The gene fragment coding for PDE5C was amplified by PCR (Polymerase Chain Reaction) using the following primers:

FW 5'-GGTTGGGAATTGCAAGAAACAAGAGAGCTACAGTCG-3'; RV 5'-GGAGATGGGAAGTCATTAGTTCCGCTTGGCCTGGCCG-3'.

The DNA fragment was then subcloned into the Histagged expression vector pLATE 52. The positive clones were sequenced and then transformed into E. coli BL21 cells (Strata-

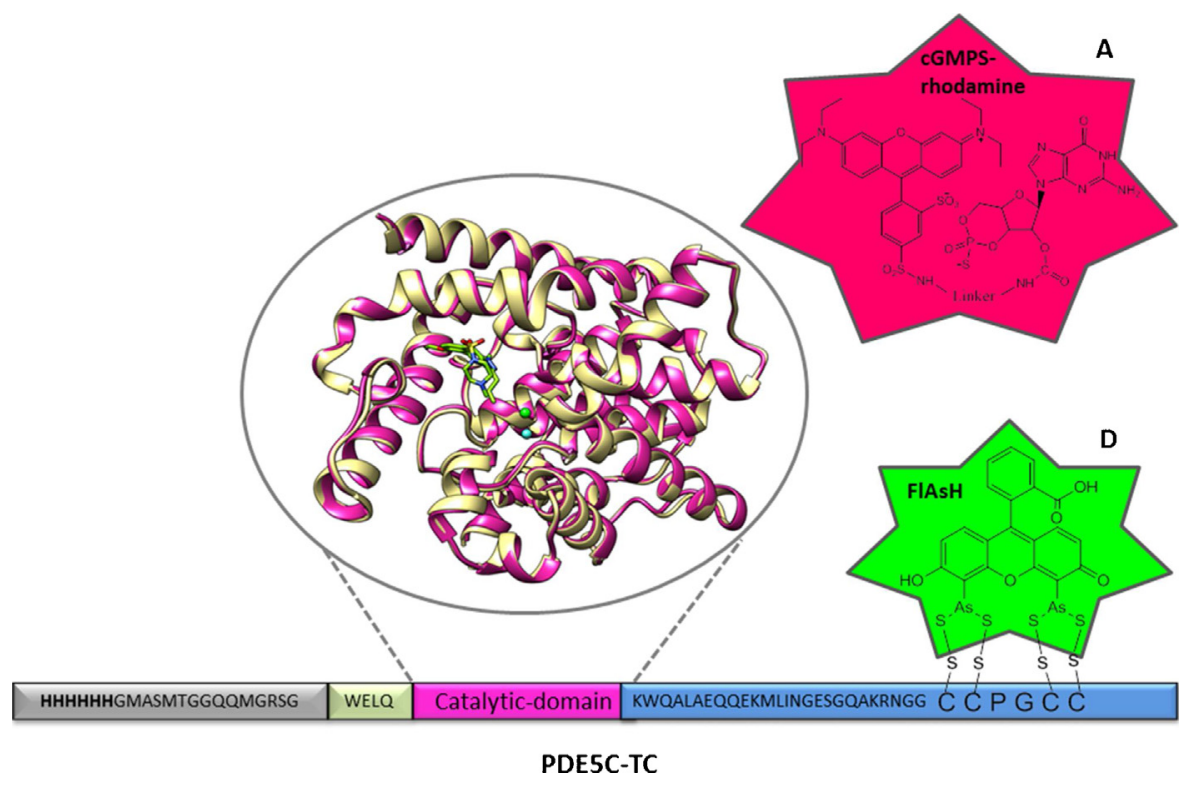

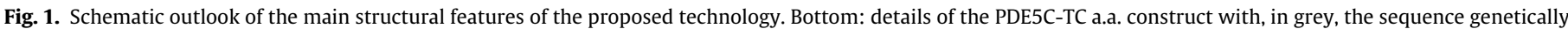

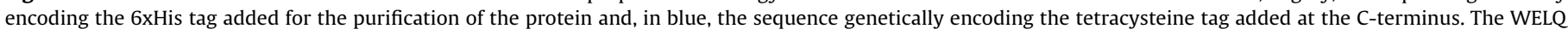

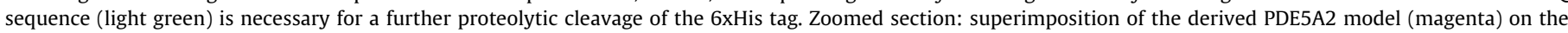

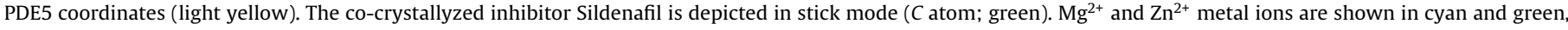

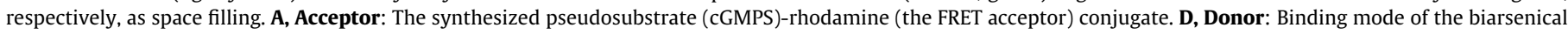
FlAsH probe to the tetracysteine tag. (For interpretation of the references to colour in this figure legend, the reader is referred to the web version of this article.) 
gene, La Jolla, CA). A second clone coding for the PDE5C-TC (the PDE5 catalytic domain with the tetracysteine tag) was obtained using the above mentioned forward primer and a new reverse primer containing the oligonucleotides triplets coding for the TC-motif (GGCCPGCCGG): RV5'- ttaACCACCACAGCAGCCCGGGCAACAGCCACCgttccgcttggcctggc $-3^{\prime}$.

The amplified fragment was then cloned into pLATE 52 vector.

The $E$. coli transformants containing the PDE5C-TC-motif were grown in enriched LB medium with $0.4 \%$ glucose and $1 \mathrm{mM} \mathrm{MgSO}_{4}$ at $37^{\circ} \mathrm{C}$, and the protein expression was induced (O.D.600 $\cong 0.8$ ) with $0.1 \mathrm{mM}$ (IPTG at $25^{\circ} \mathrm{C}$ ), overnight. Cells were harvested by centrifugation at $4500 \mathrm{rpm}$ for $10 \mathrm{~min}$ at $4{ }^{\circ} \mathrm{C}$. Crude extracts were resuspended directly in solvent $\mathrm{A}(20 \mathrm{mM}$ Tris base; $\mathrm{pH} 8.0$; $0.05 \mathrm{M} \mathrm{NaCl} ; 15 \mathrm{mM}$ imidazole; $1 \mathrm{mM} \beta$-mercaptoethanol; $1 \mathrm{mM}$ phenylmethylsulfonyl fluoride) and were purified by AKTA ${ }^{\circledR}$ FPLC (GE-Healthcare, Little Chalfont, UK) using a column HisTrap FF crude (GE Healthcare Little Chalfont, UK) for IMAC chromatography perfused at a flow rate of $3 \mathrm{ml} / \mathrm{min}$ with a mobile phase-containing solvent $A$ and a linear gradient from $0 \%$ to $100 \%$ of solvent $B(20 \mathrm{mM}$ Tris base, $\mathrm{pH} 8.0 ; 0.3 \mathrm{M} \mathrm{NaCl} 150 \mathrm{mM}$ imidazole and $1 \mathrm{mM} \beta$ mercaptoethanol) for elution of the proteins. The proteins were eluted at $98 \%$ of solvent B. For further spectrofluorometric measurements $\beta$-mercaptoethanol was replaced by $1 \mathrm{mM}$ TCEP.

The two proteins (PDE5C and PDE5C-TC) were analysed by SDSPAGE, by western blot and by mass spectrometry after tryptic digestion and quantified by Lowry's method.

\subsection{Tryptic digestion}

The pure proteins were subjected to digestion with TPCK modified sequencing grade trypsin (final enzyme to substrate ratio $1: 50 \mathrm{w} / \mathrm{w}$ ) at $37^{\circ} \mathrm{C}$ overnight. $12 \mu \mathrm{g}$ of protein extract were treated with $5 \mu \mathrm{l}$ of ammonium bicarbonate (AMBIC) $100 \mathrm{mM}$, reduced with dithiothreitol (DTT, $10 \mathrm{mM}, 1 \mu \mathrm{l}$ in AMBIC $100 \mathrm{mM}$ ) at $56^{\circ} \mathrm{C}$ for $30^{\prime}$ and alkylated with 2-iodoacetamide ( $55 \mathrm{mM}, 1 \mu \mathrm{l}$ in AMBIC $100 \mathrm{mM}$ ) at room temperature (RT) in the dark for $1 \mathrm{~h}$. The excess of alkylating agent was quenched with $10 \mu \mathrm{l}$ DTT $10 \mathrm{mM}$ at RT for $10^{\prime}$ [20]. Samples were then acidified with $5 \%$ formic acid (FA) solution and dried in a vacuum evaporator. Peptides were suspended in $30 \mu \mathrm{l}$ of $1 \% \mathrm{FA} /$ acetonitrile $98: 2$ solution and injected to a LC-ESI-QTOF mass spectrometer (Agilent Technologies, Santa Clara CA,USA). The raw data were searched against Swiss Prot database (SwissProt_57.15.fasta) using an in-house made MASCOT Server (version 2.4, Matrix Science, UK) [14].

\subsection{PDE enzymatic assay}

The activities of PDE5C and PDE5C-TC were measured with the method described by Thompson and Appleman [15] in $60 \mathrm{mM}$ Hepes pH 7.2, $0.1 \mathrm{mM}$ EGTA, $5 \mathrm{mM} \mathrm{MgCl}_{2}, 0.5 \mathrm{mg} / \mathrm{ml}$ bovine serum albumin, and $30 \mu \mathrm{g} / \mathrm{ml}$ soybean trypsin inhibitor, in a final volume of $0.15 \mathrm{ml}$. The reaction was started by adding $\left[{ }^{3} \mathrm{H}\right] \mathrm{cGMP}$ substrate at indicated concentrations. Samples were incubated at $30^{\circ} \mathrm{C}$ for $15 \mathrm{~min}$. The reaction was stopped by the addition of $0.1 \mathrm{~N} \mathrm{HCl}$ and neutralized with $0.1 \mathrm{~N} \mathrm{NaOH}$ in $0.1 \mathrm{M} \mathrm{Tris-} \mathrm{HCl} \mathrm{pH}$ 8.0. Subsequently, $2 \mathrm{mg} / \mathrm{ml}$ of $5^{\prime}$-nucleotidase (snake venum from Crotalus atrox; Sigma-Aldrich) in $0.1 \mathrm{M}$ Tris- $\mathrm{HCl}$ pH 8.0 were added. Samples were gently mixed and incubated at $30^{\circ} \mathrm{C}$ for $30 \mathrm{~min}$ to allow complete conversion of $5^{\prime}$-nucleotide to its corresponding nucleoside. Unhydrolysed cyclic nucleotide and corresponding nucleoside were separated by DEAE-Sephadex A-25 columns. The eluate was mixed with scintillation liquid ULTIMA GOLD (PerkinElmer Waltham, MA, USA) and counted on a Tri-Carb 2100TR Liquid Scintillation Counter (2000CA; Packard Instruments, Meriden, CT)]. PDE activity and $\mathrm{K}_{\mathrm{M}}$ values were expressed as mean \pm SD of three independent experiments.

\subsection{Spectroscopic measurements}

UV-vis spectra were recorded with a Varian Cary ${ }^{\circledR} 100$ UV-vis (Varian, Inc.) spectrophotometer. Fluorescence measurements were performed with a Horiba Jobin-Yvon FluoroMax3 spectrofluorometer (Horiba Scientific, Kyoto, JP). Samples were contained in $4 \times 10 \mathrm{~mm}^{2}$ quartz cuvettes. UV-vis absorption spectra for PDE5C/PDE5C-TC were recorded in the range between 230 and $350 \mathrm{~nm}$, using the $\varepsilon_{280}=38430 \mathrm{M}^{-1} \mathrm{~cm}^{-1}$ to determine the protein concentration $(800 \mu \mathrm{l}$ of a PDE5TC-FlAsH solution in $20 \mathrm{mM}$ Tris base; $\mathrm{pH} 8.0 ; 0.15 \mathrm{M} \mathrm{NaCl} ; 1 \mathrm{mM}$ TCEP). For the emission spectra, the excitation wavelength was set at $465 \mathrm{~nm}$, i.e., the wavelength where the relative contribution of the absorption of the energy transfer acceptor (rhodamine) relative to the donor (FlAsH) was minimum; the emission spectra were collected from 480 to $700 \mathrm{~nm}$. Fluorophore concentrations were determined spectrophotometrically using the following extinction coefficients: $\varepsilon_{509}$ $($ FlAsH+ FLNCCPGCCMEP $)=64000 \mathrm{M}^{-1} \mathrm{~cm}^{-1}$ and, for tetramethylrhodamine, closely related with the employed Rhodamine Red- $\mathrm{X}^{\mathrm{TM}}$ probe, $\varepsilon_{574}$ (tetramethylhodamine) $=85000 \mathrm{M}^{-1} \mathrm{~cm}^{-1}$.

All measurements were performed, in $20 \mathrm{mM}$ Tris base, $\mathrm{pH} 7.5$, plus $0.15 \mathrm{M} \mathrm{NaCl}$, and $1 \mathrm{mM}$ TCEP, and were carried out at room temperature $\left(22 \pm 2{ }^{\circ} \mathrm{C}\right)$.

\section{6. cGMPS-rhodamine synthesis}

cGMPS-rhodamine crude product was purified by preparative reversed-phase HPLC using a Water Delta Prep 3000 system with a Waters PrepLC $40 \mathrm{~mm}$ Assembly column $\mathrm{C}_{18}(30 \times 4 \mathrm{~cm}, 300 \AA$, $15 \mu \mathrm{m}$ spherical particle size column). The column was perfused at a flow rate of $2 \mathrm{ml} / \mathrm{min}$, with a mobile phase-containing solvent $\mathrm{A}$ $\left(100 \% \mathrm{v} / \mathrm{v}\right.$ of $\mathrm{H}_{2} \mathrm{O} ; 0.1 \% \mathrm{v} / \mathrm{v}$ TFA), and a linear gradient from $0 \%$ to $100 \%$ of solvent $\mathrm{B}\left(60 \% \mathrm{v} / \mathrm{v}\right.$ of $\mathrm{CH}_{3} \mathrm{CN} ; 40 \% \mathrm{v} / \mathrm{v} \mathrm{H}_{2} \mathrm{O} ; 0.1 \% \mathrm{v} / \mathrm{v}$ TFA) in $25 \mathrm{~min}$ was used for elution of the compound. HPLC analysis was performed using a Beckman System Gold with a Hypersil BDS C18 column ( $5 \mu \mathrm{m} ; 4.6 \times 250 \mathrm{~mm})$. Analysis was performed with the solvent system reported above programmed at flow rates of $1 \mathrm{ml} / \mathrm{min}$ and with a linear gradient from $0 \% \mathrm{~B}$ to $100 \% \mathrm{~B}$ in $25 \mathrm{~min}$. To a stirred solution of RP-2'-AHC-cGMP $(0,005 \mathrm{mmol})$ in a $1: 1 \mathrm{mix}-$ ture of $\mathrm{CH}_{3} \mathrm{CN} / \mathrm{H}_{2} \mathrm{O}(1 \mathrm{ml})$, rhodamine Red-X $\mathrm{X}^{\mathrm{TM}}(0.005 \mathrm{mmol})$ and DIPEA $(0.01 \mathrm{mmol})$ were added. The fluorescent violet solution was stirred in the dark at room temperature for $30 \mathrm{~min}$. After this time, analytical HPLC showed a total conversion of the starting materials. The reaction mixture was directly injected in preparative HPLC giving the desired cGMPS-rhodamine as a violet solid (53\% yield) after lyophilisation (analytical HPLC and mass spectra are available as Supplementary material Fig. S1). cGMPS-rhodamine showed $>95 \%$ purity and its molecular weight $\left([\mathrm{M}+\mathrm{H}]^{+} 1157,3509 \mathrm{~g} / \mathrm{mol}\right)$ determined by electrospray ionisation (ESI) (MICROMASS ZMD 2000, Waters Alliance, Milford, MA, USA) resulted in line with the expected molecular formula $(1156,27 \mathrm{~g} / \mathrm{mol})$.

\subsection{In silico evaluation of PDE5 - cGMPS-rhodamine interaction}

The chemical structure of cGMPS-rhodamine was built, parameterized (Gasteiger-Hückel method) and energy minimized within Sybyl-X 1.0 using the Tripos force field [16]. PDE5A2 has been modelled on the basis of the PDE5 X-ray data in complex with Sildenafil, downloaded from the protein databank (pdb code 1TBF; resolution $=1.30 \AA$ ) [17]. The sequence alignment was derived on the basis of the Blosum62 matrix by means of the MOE software [18]. The final homology model was derived using MOE, following a procedure previously adopted for other case studies [19].

A thorough evaluation of the final PDE5A2 model was performed using Ramachandran plots, and also exploring the contact energy values and the rotamer profile by a comparison with 
those evaluated for the X-ray template. Docking studies of cGMPSrhodamine have been performed by means of the Surflex docking module implemented in Sybyl-X1.0. Surflex-Dock uses an empirically derived scoring function based on the binding affinities of protein-ligand complexes and on their X-ray structures. This kind of parameter is a weighted sum of non-linear functions involving van der Waals surface distances between the appropriate pairs of exposed protein and ligand atoms, including the hydrophobic, polar, repulsive, entropic and solvation and crash terms. Finally, the derived protein-ligand complex stability was assessed using a short molecular dynamics (MD), $\sim 1$ ps run, at constant temperature, followed by an all-atom energy minimization (LowModeMD implemented in MOE software), as some of us previously discussed in other homology modelling-based studies [20]. All calculations were carried out on a standard personal computer running under Windows XP.

\subsection{PDE5-TC labeling}

The labelling of PDE5C-TC with FlAsH was obtained by incubating the protein $(20 \mu \mathrm{M})$ with 1.5 equivalents of FlAsH-EDT 2 (Life Techonologies Carlsbad, CA, USA) in $20 \mathrm{mM}$ TRIS, $0.15 \mathrm{M} \mathrm{NaCl}$, $1 \mathrm{mM}$ TCEP, $\mathrm{pH} 8$ for $1 \mathrm{~h}$ at room temperature in the dark [8]. The reaction mixture was applied to a G25 Sephadex (GE Healthcare, Little Chalfont, Buckinghamshire, UK) column material to remove the unbound FlAsH-EDT 2 . The eluted Protein-FlAsH complex was checked by UV-vis and SDS-PAGE (12\% acrylamide under reducing conditions) [9].

\subsection{FRET and displacement experiments}

The non-radiative energy transfer (FRET) between the PDE5CTC-bound excited donor (FlAsH) and the cGMPS-bound rhodamine acceptor was measured using a Horiba Jobin-Yvon FluoroMax3 spectrofluorometer (Horiba Scientific, Kyoto, JP). The FRET efficiency, that is proportional to the fraction of PDE5C-TC-FlAsH bound to cGMPS-rhodamine, was determined by measuring the emission intensities of the FlAsH donor $\left(\mathrm{I}_{\mathrm{F}}\right)$ at $530 \mathrm{~nm}$ and the rhodamine acceptor at $600 \mathrm{~nm}\left(\mathrm{I}_{\mathrm{R}}\right)$ following progressive cGMPSrhodamine additions. The fluorescence spectra were obtained at $\lambda_{\text {exc }}=465 \mathrm{~nm}$, a wavelength selected in order to afford the maximum $I_{F} / I_{R}$ relative excitation. All spectroscopic experiments were carried out in $20 \mathrm{mM}$ Tris base, $0.15 \mathrm{M} \mathrm{NaCl}$, and $1 \mathrm{mM}$ TCEP at the carefully controlled $\mathrm{pH}=7.5$. Data analysis and the determination of the $\mathrm{K}_{\mathrm{d}}$ value for the PDE5C-TC-FlAsH/cGMPS-rhodamine complex, hereafter called 'complex 1', are described in detail in the results section.

To investigate the decrease in FRET efficiency due to displacement of the pseudosubstrate upon competitive inhibitor binding, solutions of tadalafil and cGMPS were progressively added to the complex 1 solutions and the $\mathrm{I}_{\mathrm{F}} / \mathrm{I}_{\mathrm{R}}$ (donor/acceptor) intensity ratio value was analyzed as a function of the added inhibitor concentrations. Typical protein cGMPS-rhodamine concentrations were between 5 and $20 \mu \mathrm{M}$.

Data handling and $\mathrm{K}_{\mathrm{d}}$ calculation for the competitor are based on the original articles by Wang and co-workers, in which the classical quadratic equation becomes cubic due to the presence of three components in solution, namely the two ligands (cGMPSrhodamine and inhibitor) and the protein, generating a three-state equilibrium binding model $[21,22]$. We chose to compute the concentration of complex 1 ('PA' in Wang's expressions) in terms of the ratio of the $530 \mathrm{~nm}\left(I_{F}\right)$ to $600 \mathrm{~nm}\left(I_{R}\right)$ emission intensities, $R$, using Eq. (1):

$R=\frac{R^{\infty}(1-f)}{1+R^{\infty} \cdot a \cdot f}$ where $R^{\infty}$ is the value of $R$ at the end of the displacement, $\mathrm{f}=$ [complex 1]/[PDE $]_{\text {tot }}$ is the fraction of protein bound to cGMPSrhodamine and $\mathrm{a}=\Phi_{\mathrm{R} \alpha \mathrm{R}} / \Phi_{\mathrm{F} \alpha \mathrm{F}}$, with $\Phi_{\mathrm{R}, \mathrm{F}}$ indicating the emission quantum yields of the two probes and $\alpha_{R, F}$ the instrumental sensitivities of the employed detection channel at $600 \mathrm{~nm}(\mathrm{R})$ and $530 \mathrm{~nm}(\mathrm{~F})$. We estimated this parameter to hold 0.5 in our conditions. A derivation of Eq. (1) is given in the Supplementary information (Expression of the donor/acceptor emission intensity ratio in terms of the concentration of complex 1 ). Wang's equation (Eq. (14) in ref. [21]) was then employed to compute the concentration of complex 1 (PA according to Wang) in terms of the known total concentrations of the protein and of the two competing ligands and the dissociation constants of the two complexes. The $\mathrm{R}$ values computed this way were visually compared with the experimental $R$ values, thus enabling us to estimate the $K_{d}$ value for the complex (complex 2 ) with the incoming ligand ( $K_{B}$ in Wang's treatment).

\section{Results and discussion}

\subsection{Protein characterization}

The PDE5A gene encodes three isoforms (PDE5A1, PDE5A2 and PDE5A3), generated through alternative splicing of the first exon of the PDE5A gene. These isoforms share the same C-terminal catalytic domain (C), differing only in the N-terminal portion. In this work, the sequence corresponding to the $\mathrm{C}$-terminal catalytic domain of PDE5A2 (aa 536-860) was cloned in two different forms: the wt (PDE5C) and the tetracysteine-tagged catalytic domain (PDE5CTC). The TC (CCPGCC) motif was successfully introduced at the C-terminus of the PDE5C domain by PCR. Insertion was confirmed by DNA sequencing. The proteins were expressed in $-E$. coli and successfully purified by a two-step purification procedure leading to more than $90 \%$ of pure product. The purified proteins were analyzed by ESI Q-TOF MS to assess protein identity. The pure proteins were digested with trypsin and analyzed by ESI Q-TOF MS-MS yielding $80 \%$ sequence coverage with the human PDE5A2 catalytic domain.

The purified proteins were tested for enzymatic activity using $1 \mu \mathrm{M}\left[{ }^{3} \mathrm{H}\right] \mathrm{CGMP}$ as the substrate. PDE5C and PDE5C-TC were similarly active with $0.17 \pm 0.04$ and $0.18 \pm 0.07 \mu$ moles of cGMP hydrolised $/ \mathrm{min} / \mathrm{mg}$, respectively. These data are in accordance with the activity of full length PDE5 [23] and demonstrate that dimerization is not required for PDE5 catalytic activity, as both recombinant catalytic domains were monomeric [24,25]. Moreover the Michaelis constant for PDE5C-TC was determined (Fig. S2 Supplementary information): $\mathrm{K}_{\mathrm{M}}=10.7 \pm 1.4 \mu \mathrm{M}$. This value is similar to that obtained with the purified full length enzyme [2].

The SDS-PAGE analysis of PDE5C and PDE5C-TC-FIAsH outlined the presence of the green fluorescent bands in lanes containing the PDE5C-TC-FlaAsH adductconfirming the labelling of the PDE5CTC [9]. The presence of such fluorescent bands in the gel obtained under reducing conditions confirms the covalent character of the bonding of FlAsH to PDE5C-TC. Unspecific labelling was negligible under our experimental conditions. (Fig. S3-Supplementary information). Moreover, the covalent binding of FlAsH did not modify the kinetic properties of the enzyme. Indeed, the $\mathrm{K}_{\mathrm{M}}$ of PDE5CTCFlAsH, $12.8 \pm 2.2 \mu \mathrm{M}$, was not significantly different from that of PDE5C-TC (Fig. 2 Supplementary information).

Formation of the PDE5C-TC-FlAsH adduct was indicated in the UV-vis absorption spectrum by the appearance of a band typical of cysteinebound to FlaAsH with maximum at $509 \mathrm{~nm}$ (Fig. S4Supplementary information), and was confirmed by the appearance of a fluorescence emission band with maximum at $530 \mathrm{~nm}$ whose intensity increased with time for $30-40 \mathrm{~min}$ in agree- 
ment with the observed behaviour of other FlAsH complexes [26]. In order to remove residual traces of nickel ions bound to the tetracysteine-tag during the IMAC chromatography, the protein was treated with $0.5 \mathrm{mM}$ EDTA before labelling it. Additionally, because of the FlAsH-EDT 2 large excess, emission from unbound tag and radiative transfer to the rhodamine acceptor would interfere in subsequent experiments aiming at determining FRET efficiencies. To remove the unbound FlAsH-EDT 2 we passed the protein+probe solution through a desalting chromatography column. This treatment, together with the pre-treatment with EDTA, led to a spectrophotometrically determined (bands at 280 and $509 \mathrm{~nm}$ in Fig. S4) 1:1 PDE-TC/FlAsH concentration ratio, and caused an increase in the observed FRET efficiency by a factor of 10.

\subsection{PDE5 homology modeling and docking studies}

We first discuss the PDE5A2 homology model derived from the X-ray crystallographic data of the available PDE5 in order to explore the putative binding mode of the fluorescent ligand cGMPSrhodamine through docking calculations. This approach expected to yield some valuable, although preliminary information, for the design of novel ligands.

The PDE5A2 homology model was built following the procedure that some of us recently reported for other PDEs [19]. Briefly, the amino acidic sequence of the PDE5A2 catalytic site was aligned with that of the PDE5 X-ray structure (pdb code: 1TBF; resolution $=1.30 \AA$ ) [17] (Fig. S5 Supplementary information). The reliability of the alignment demonstrated by the high value of the pairwise percentage residue identity calculated between the two proteins, $\mathrm{PPRI}=93.3 \%$.

Then, the derived PDE5A2 model was superimposed on the coordinates of PDE5 (Fig. S10 Supplementary information), used as a template, and a good $\mathrm{C}$-atom aligment root-mean-square deviation value was obtained $(\mathrm{RMSD}=0.456 \AA$ ). The geometry and the related backbone conformation of the model, inspected by Ramachandran plot (see Fig. S6, Supplementary information), showed no outliers, thereby yielding a good validation of the computational protocol. In addition, the structural reliability of the model has been evaluated by comparing the energy profiles of the derived PDE5A2 catalytic site and of the template, PDE5. In general, high negative values and positive energy terms correspond to residues which are expected to be oriented toward a hydrophobic and a hydrophilic environment, respectively. Notably, the observed energetic profile of the two proteins proved to follow the same polar trend (see Fig. S7, Supplementary information). Also, model quality estimation for the protein side-chains was performed by the rotamer energy profile; this showed no outliers, since all the residues fell within the recommended energy values (Fig. S8, Supplementary information).

Taken together, these data validate the PDE5A2 model. This was then employed to explore the putative binding mode of cGMPS-rhodamine. For this kind of analysis, we considered that the enzymes included in the PDE5A family are characterized by highly conserved pockets classified as a metal-binding pocket ( $M$ pocket), a solvent-filled side pocket (S pocket) and a third region known as hydrophobic clamp ( $Q$ pocket). These cavities include: (i) H613, H617, H653, D654, H657, N662, L681, E682, D724, L725, D764 (M pocket); (ii) G659, N662, E785, F786, Q789, T802, M805 (S pocket); (iii) Y612, L765, A767, I768, Q775, I778, A779, V782, A783, L804, I813, M816, Q817, F820 (Q pocket), as described for the PDE5 isoform [27]. The results reported by Card [27], as well as a number of crystallographic data about PDE5 complexes, highlight the key role played by a glutamine residue in the $\mathrm{Q}$ pocket engaged in the ligand binding. Accordingly, the inhibitor Sildenafil co-crystallized in the PDE5 catalytic site of 1TBF employed in this work, exhibits two H-bonds with Q817 (see Fig. S9, Supplementary information). Based on this information and on a superimposition of the obtained PDE5A2 on the X-ray structure of PDE5, we outlined the corresponding binding site of the modelled protein, in order to proceed with docking calculations of cGMPS-rhodamine. Our results revealed a conserved $\mathrm{H}$-bond with the aforementioned glutamine residue Q817 thanks to the purine cycle that exhibits a position similar to that of Sildenafil (inhibitor crystallized with PDE5), while the dioxaphosphinine substituent properly moves towards the metal pocket. Conversely, the bulky rhodamine portion proved to lay outside the enzyme cavity (Fig. 2).

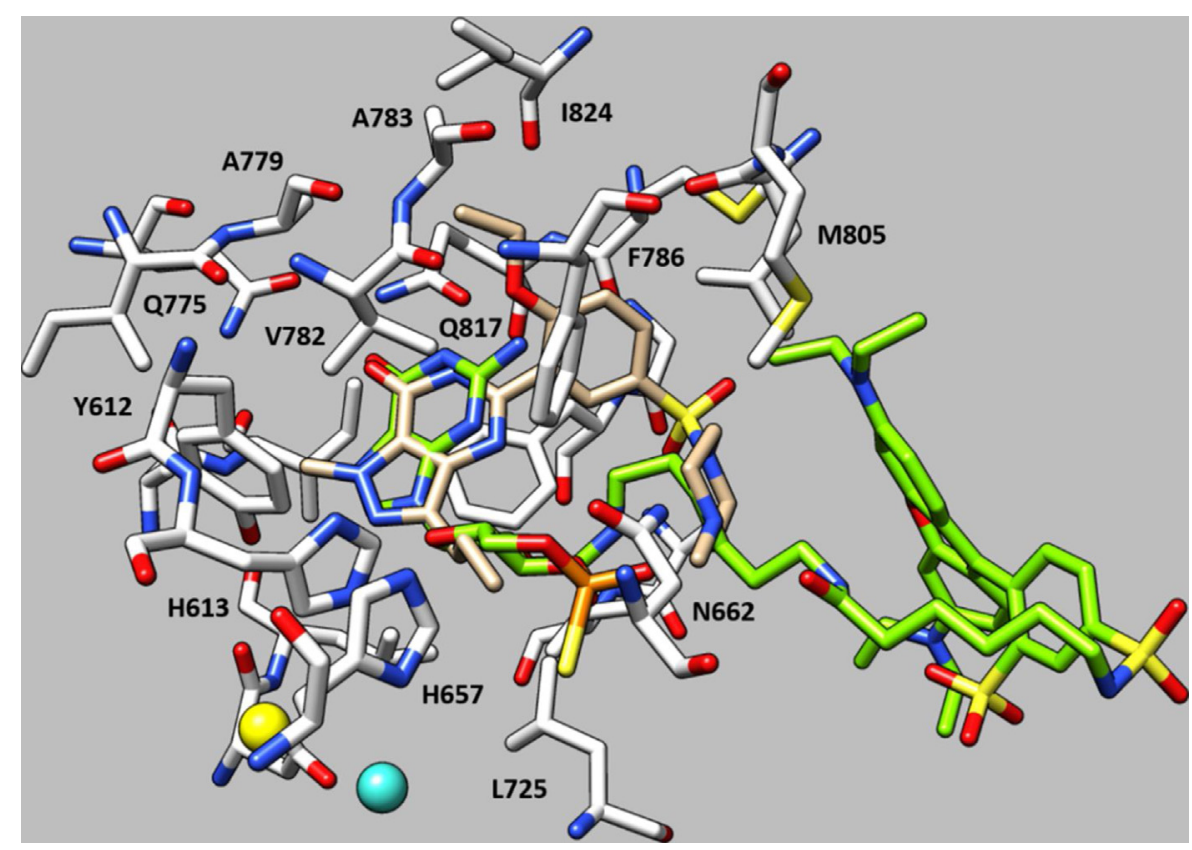

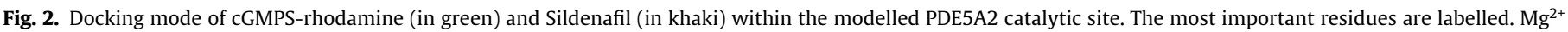

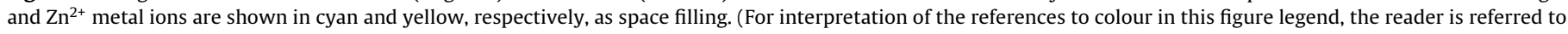
the web version of this article.) 

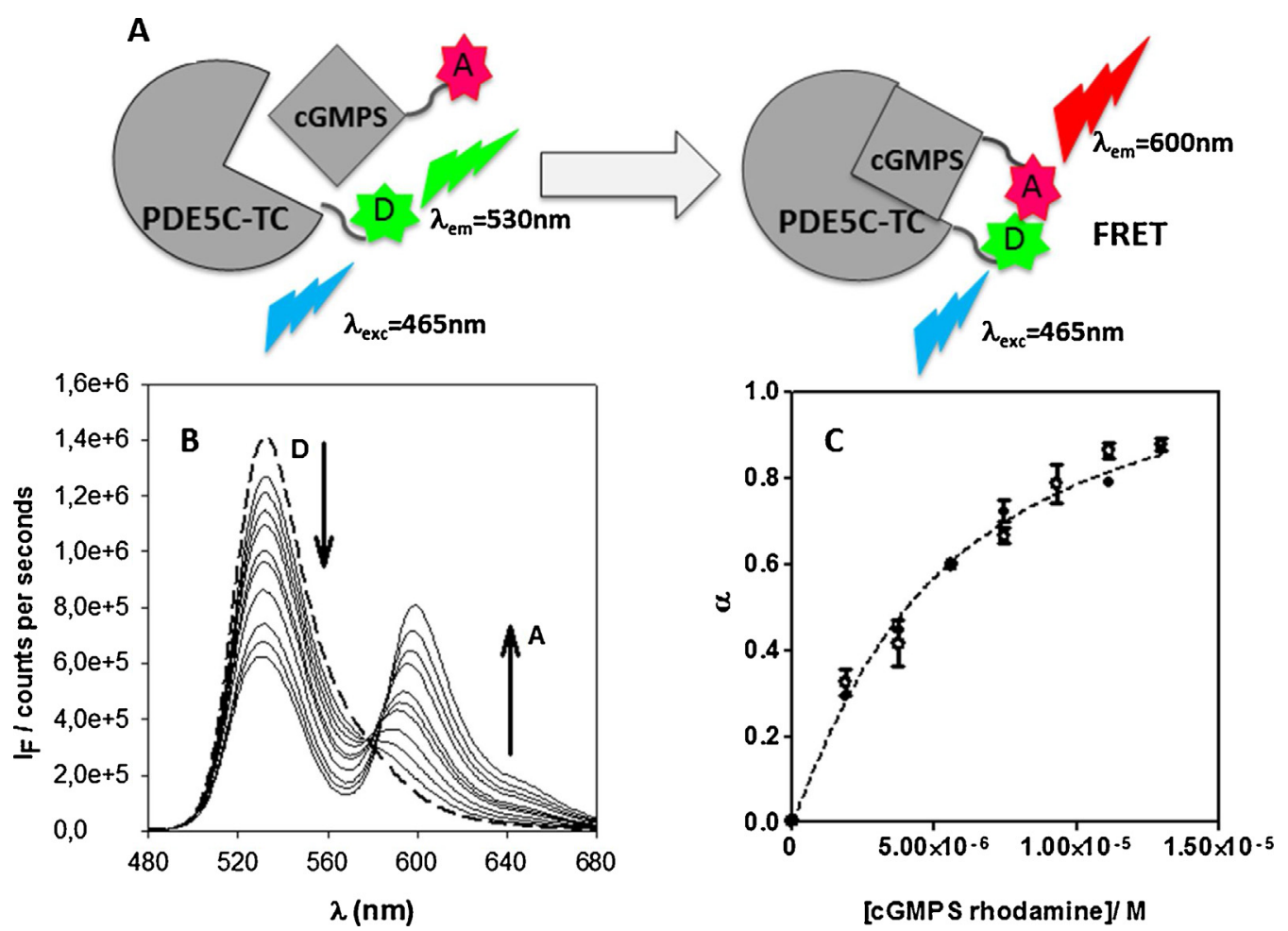

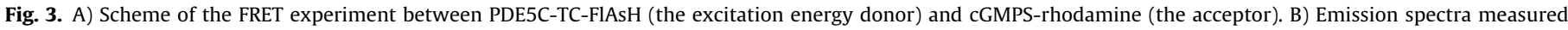

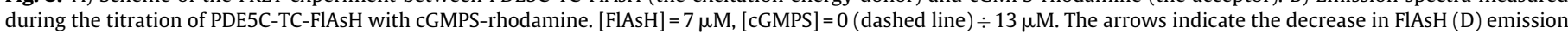

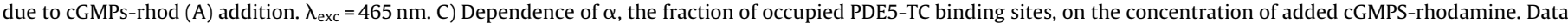

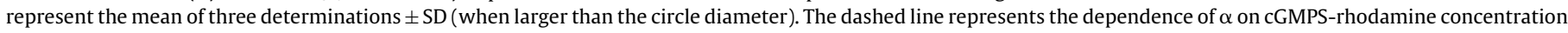
computed with the best-fitting value of $\mathrm{K}_{\mathrm{d}}$.

\subsection{Binding equilibrium between PDE5C-TC and cGMPS-rhodamine}

Förster resonance energy transfer (FRET), the non-radiative transfer of electronic excitation energy, has become widely employed to study protein-protein and protein-ligand interactions $[28,29]$. The FRET efficiency results from coupling between the donor and the acceptor transition dipoles and, therefore, depends on their relative orientation and decreases with the sixth power of their distance [29]. Additionally, it depends on the spectral overlap between the emission spectrum of the donor and the absorption spectrum of the acceptor. For a given donor/acceptor pair, the FRET efficiency is synthesized by the value of the Förster critical distance, i.e., the distance at which the FRET efficiency is 0.5 [29]. In this paper, we study the interaction between the catalytic domain of PDE5C-TC, made fluorescent by binding of FlAsH, the energy donor (D), at the tetracysteine motif and the pseudosusbtrate cGMPS made fluorescent by conjugation to rhodamine (the acceptor, A). We developed a synthesis of cGMPS-rhodamine that readily allowed access to milligram quantities of this compound. FRET was then evaluated by measuring the intensity of the donor $\left(I_{F}\right)$ in the absence and in the presence of the acceptor. To facilitate an understanding of the method, the rationale of the FRET experiment is summarised in the cartoon in Fig. 3A.

To generate equilibrium binding curves, PDE5C-TC-FlAsH was titrated with increasing amounts of cGMPS-rhodamine. Preferential excitation of FlAsH $\left(\lambda_{\text {exc }}=465 \mathrm{~nm}\right)$ produced emission by FlAsH, with maximum at $530 \mathrm{~nm}$, and triggered excitation energy-transfer to rhodamine and subsequent emission from latter with maximum at $600 \mathrm{~nm}$. The occurrence of $\mathrm{FlAsH}(\mathrm{D})$-to-rhodamine(A) FRET in PDE5C-TC-FlAsH/cGMPS-rhodamine complexes was demonstrated by the decrease of the $\mathrm{D}$ emission band at $530 \mathrm{~nm}$ and the concomi- tant growth of the A emission band at $600 \mathrm{~nm}$ following addition of cGMPS-rhodamine to a PDE5C-TC-FlAsH solution (Fig. 3B).

The observed $\mathrm{I}_{530}$ intensities $\left(\mathrm{I}_{\mathrm{obs}}\right)$ were corrected in order to account for the increasing absorbances of the added rhodamine at the excitation and emission wavelengths $\left(A_{465}\right.$ and $\left.A_{530}\right)$ as follows:

$I_{530}=I_{o b s} 10^{\left(A_{465}+A_{530}\right) / 2}$

and were used to determine the fraction of occupied binding sites, $\alpha$, in the PDE5-TC enzyme. This was then plotted as a function of the concentration of added cGMPS-rhodamine. In the non-linear least squares regression analysis based on the standard Hill Eq. (3) a value of $\mathrm{n} \cong 1$ was obtained, thus confirming a single binding site for the substrate. (Fig. 3C).

$$
\begin{aligned}
& \frac{[\text { PDE5C }- \text { TC }- \text { FlAsH } / \text { cGMPS }- \text { rhodamine }]}{[\text { PDE5C }- \text { TC }- \text { FlAsH }]_{\text {tot }}} \\
& =\alpha=\frac{I_{\text {max }}-I}{I_{\max }-I_{0}}=\frac{[\text { cGMPS }- \text { rhodamine }]^{n}}{K_{d}+[\text { cGMPS }- \text { rhodamine }]^{n} \text { tot }}
\end{aligned}
$$

In Eq. (3), $K_{d}$ is the apparent dissociation constant. $I_{\max }$ and $I_{0}$ are the maximum and the minimum values of the $\mathrm{I}_{530}$, respectively.

The value of $\mathrm{K}_{\mathrm{d}}$, obtained, $11.5 \pm 1.5 \mu \mathrm{M}$, is representative of a noncovalent reversible interaction between the two molecules, and is consistent with the $\mathrm{K}_{\mathrm{M}}$ value of $10.7 \mu \mathrm{M}$ obtained for $\left[{ }^{3} \mathrm{H}\right] \mathrm{cGMP}$ (see paragraph 3.1), which is in turn comparable with published data [2].

\subsection{Competitive displacement experiments}

A competitive displacement experiment based on the equilibrium model and the above-established FRET-based technology was then developed to test PDE5 inhibitor candidates and estimate their 

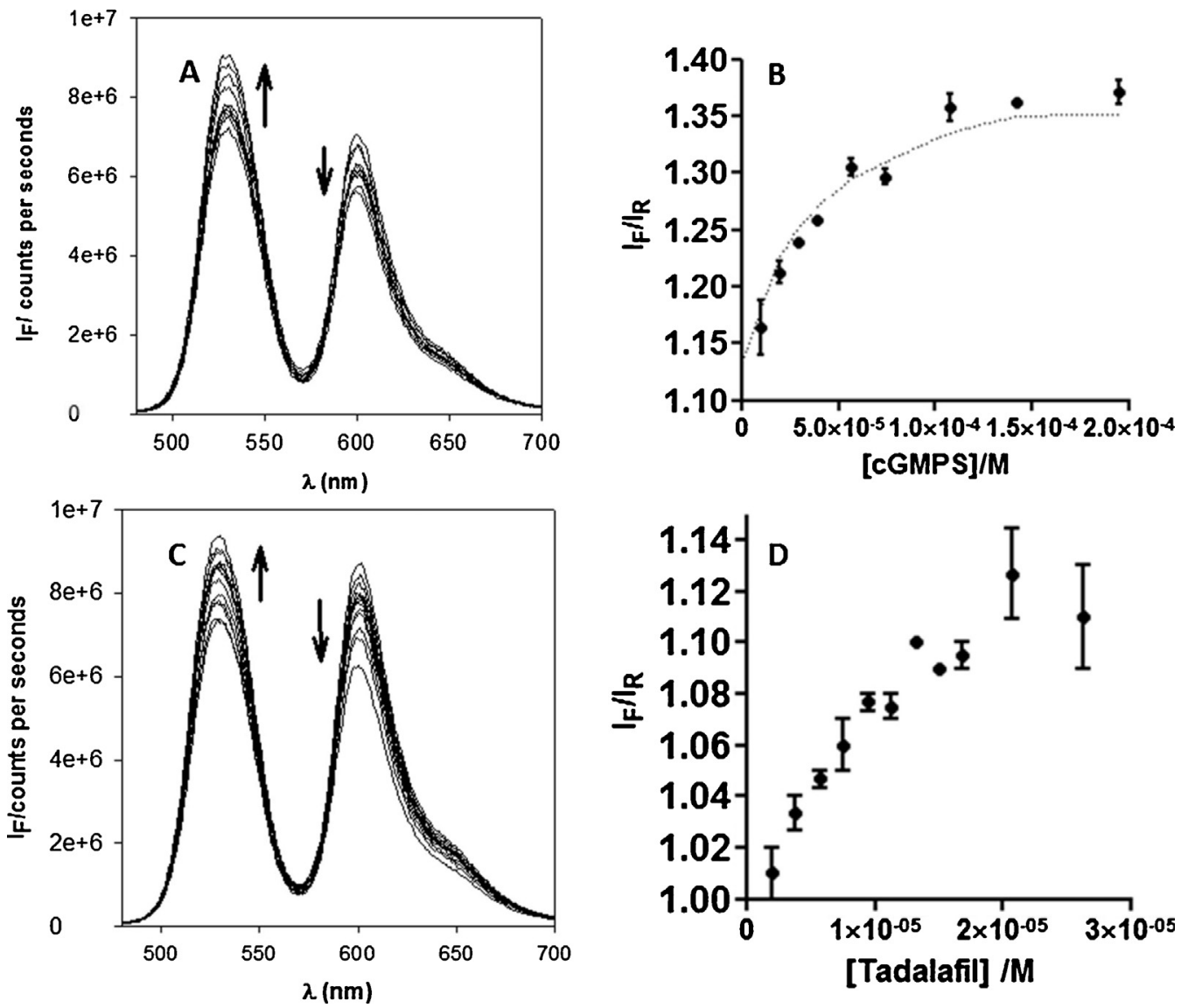

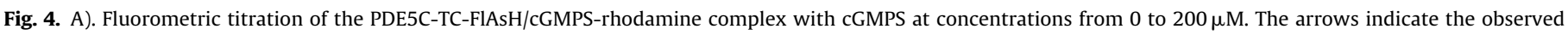

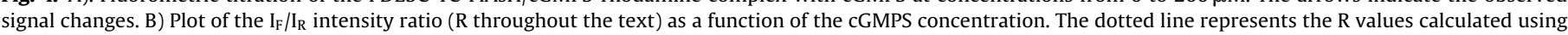

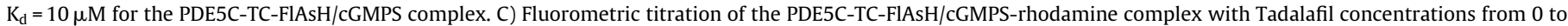

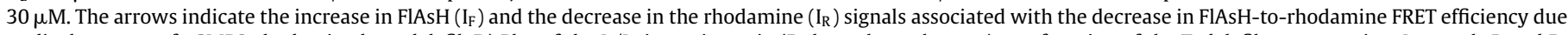

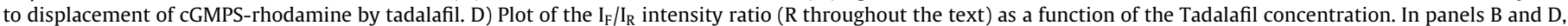
each circle represents the mean of three determinations \pm SD.

affinities. Since the $K_{d}$ of the PCE5C-TC-FlAsH/cGMPS-rhodamine (complex 1) has been obtained, the $K_{d}$ of a competing inhibitor can be estimated by measuring the relative occupancy of PDE5A by cGMPS-rhodamine and the inhibitor. This can be accomplished by evaluating the decrease in FlAsH-to-rhodamine FRET efficiency. In order to increase the sensitivity of our approach, we exploited both the expected experimental effects of the displacement, i.e., the decrease in acceptor emission and the recovery of donor emission, by computing the ratio of the $530 \mathrm{~nm}\left(\mathrm{I}_{\mathrm{F}}\right)$ to $600 \mathrm{~nm}\left(\mathrm{I}_{\mathrm{R}}\right)$ emission intensities, $\mathrm{R}$ in Eq. (1) of paragraph 2.9, as a function of the inhibitor concentration.

Complex 1 was obtained by combining $30 \mu \mathrm{M}$ cGMPSrhodamine and $5 \mu \mathrm{M}$ PDE5C-TC-FlAsH. At these concentrations, the fraction of occupied PDE5C sites, and the FRET efficiency, are 0.7. Complex 1 was then titrated with increasing amounts of catalyticsite ligands.

We tested this approach with two ligands that exhibit widely differing affinities for the binding pocket of PDE5C, namely cGMPS (presumably 10-12 $\mu \mathrm{M}$ ) and tadalafil (nanomolar).

cGMPS was added to complex 1 at concentrations from 0 to $200 \mu \mathrm{M}$. We observed an increase in the donor band intensity $\left(\mathrm{I}_{\mathrm{F}}\right)$ together with a decrease in the acceptor band intensity $\left(\mathrm{I}_{R}\right)$ (Fig. $4 \mathrm{~A}$ ), indicating that cGMPS-rhodamine was displaced at the PDE5C-TC binding site by cGMPS.The measured intensities are displayed, as the $I_{F} / I_{R}$ ratio, $R$, against the inhibitor concentration in Fig. $4 B$. As outlined in paragraph 2.9, we then compared the experimental $\mathrm{R}$ values with the R values calculated using Eq. (1), where the com- plex 1 concentrations were obtained from the Wang corresponding equation using tentative values for the equilibrium dissociation constant of complex $2[21,22]$. The value of the dissociation constant for the complex of the protein with the incoming ligand, cGMPS thus estimated, $\mathrm{K}_{\mathrm{d}}=10 \pm 2 \mu \mathrm{M}$, is consistent with the corresponding $K_{d}$ value of complex 1 and the cGMP substrate $K_{M}$ value.

To test the method with a higher-affinity ligand, Tadalafil was added to complex 1 at concentrations from 0 to $30 \mu \mathrm{M}$. Again, we observed a recovery in the donor emission intensity together with a decrease in the acceptor emission intensity, indicating displacement of cGMPS-rhodamine from complex 1 due to formation of complex 2 with Tadalafil (Fig. 4C). The $I_{F} / I_{R}$ fluorescence intensity ratios showed a behaviour consistent with quantitative binding of Tadalafil to the protein, in agreement with the published nanomolar affinity [30].

\section{Conclusion}

The FRET-based approach described here proved to be a valuable tool for detecting binding of small molecules to the catalytic pocket of PDE5A. The approach is based on competitive cuvettebased displacement experiments. The observables are the emission intensities of an electronic excitation energy pair, namely, as the donor, the fluorescein-based diarsenical probe, FlAsH, that binds proteins that incorporate a CCPGCC motif and, as the acceptor, rhodamine, that is covalently bound to a non-reactive substrate analogue of this enzyme, cGMPS. Computational work proved the 
cGMPS-rhodamine conjugate thus obtained to bind the catalytic pocket of the enzyme similarly to the natural substrate and provided some structural hints for the design of competitive inhibitors of the PDE5 enzymes. Because the $K_{d}$ value of the pseudosubstrate ligand is $10 \mu \mathrm{M}$, this displacement experiment is expected to recognize positives among compounds with $\mathrm{K}_{\mathrm{d}}$ values for PDE5C from tens of micromolar to nanomolar.

The approach is suitable for being adapted to a 96-well plate format to be employed for medium-to-high throughput screenings of new PDE5 competitive inhibitors, and for estimating their potency. Removal of the excess FlAsH donor markedly increased the observed FRET efficiency in our experiments and is thus expected to result in a sensitive and robust assay. Extension of this approach to cellular environments, following cloning of the PDE5C-TC construct into a mammalian vector, is also easily foreseen and presently underway in our laboratory.

Recent advances make this an exciting time for the research on PDEs. In particular, these enzymes hold great promise as drug targets. We believe that this new fluorescence-based tool can be translated into a simple and robust assay for screening PDE bindingpocket ligand libraries. To our knowledge this would be the only such non-radiometric screening method available today.

\section{Acknowledgments}

The authors are grateful to Dr. Federica Barbagallo for the expression vector of full-length human PDE5A2 and Dr. Domenico D'Arca for helpful discussions on the tetracys tag cloning at the C-terminal of PDE5C.

This work was supported by MIUR, Ministero dell'Istruzione, dell'Università e della Ricerca [FIRB grant; project RBFR12FI27_005 to Giulia Di Rocco]; and by Fondo di Ateneo per la Ricerca [FAR2015 to Giulia Di Rocco].

\section{Appendix A. Supplementary data}

Supplementary data associated with this article can be found, in the online version, at https://doi.org/10.1016/j.jpba.2017.11.014.

\section{References}

[1] M.F. Azevedo, F.R. Faucz, E. Bimpaki, A. Horvath, I. Levy, R.B. de Alexandre, F. Ahmad, V. Manganiello, C.A. Stratakis, Clinical and molecular genetics of the phosphodiesterases (PDEs), Endocr. Rev. 35 (2014) 195-233, http://dx.doi. org/10.1210/er.2013-1053.

[2] A.T. Bender, Cyclic nucleotide phosphodiesterases: molecular regulation to clinical use, Pharmacol. Rev. 58 (2006) 488-520, http://dx.doi.org/10.1124/pr. 58.3.5.

[3] D.H. Maurice, H. Ke, F. Ahmad, Y. Wang, J. J.Chung, V.C. Manganiello, Advances in targeting cyclic nucleotide phosphodiesterases, Nat. Rev. Drug Discov. 13 (2014) 290-314, http://dx.doi.org/10.1038/nrd4228.

[4] B. Zhu, S.J. Strada, The novel functions of cGMP-specific phosphodiesterase 5 and its inhibitors in carcinoma cells and pulmonary/cardiovascular vessels, Curr. Top. Med. Chem. 7 (2007) 437-454.

[5] P.F. Windham, P.F. Windham, cGMP signaling as a target for the prevention and treatment of breast cancer, Semin. Cancer Biol. 31 (2015) 106-110, http://dx.doi.org/10.1016/j.semcancer.2014.06.006.

[6] M. Sponziello, A. Verrienti, F. Rosignolo, R.F. De Rose, V. Pecce, V. Maggisano, C. Durante, S. Bulotta, G. Damante, L. Giacomelli, C.R.T. Di Gioia, S. Filetti, D. Russo, M. Celano, PDE5 expression in human thyroid tumors and effects of PDE5 inhibitors on growth and migration of cancer cells, Endocrine 50 (2015) 434-441, http://dx.doi.org/10.1007/s12020-015-0586-x.

[7] N. Marino, J.W. Collins, C. Shen, N.J. Caplen, A.S. Merchant, Y. Gökmen-Polar, C.P. Goswami, T. Hoshino, Y. Qian, G.W. Sledge, P.S. Steeg, Identification and validation of genes with expression patterns inverse to multiple metastasis suppressor genes in breast cancer cell lines, Clin. Exp. Metastasis 31 (2014) 771-786, http://dx.doi.org/10.1007/s10585-014-9667-0.

[8] C. Hoffmann, G. Gaietta, M. Bünemann, S.R. Adams, S. Oberdorff-Maass, B. Behr, J.P. Vilardaga, R.Y. Tsien, M.H. Ellisman, M.J. Lohse, A FlAsH-based FRET approach to determine $G$ protein-coupled receptor activation in living cells, Nat. Methods 2 (2005) 171-176, http://dx.doi.org/10.1038/nmeth742.

[9] S.R. Adams, R.E. Campbell, L.A. Gross, B.R. Martin, G.K. Walkup, Y. Yao, J. Llopis, R.Y. Tsien, New biarsenical ligands and tetracysteine motifs for protein labeling in vitro and in vivo: synthesis and biological applications, J. Am. Chem. Soc. 124 (2002) 6063-6076.

[10] B.A. Griffin, S.R. Adams, R.Y. Tsien, Specific covalent labeling of recombinant protein molecules inside live cells, Science 281 (1998) 269-272.

[11] S.C. Alexander, A. Schepartz, Interactions of AsCy3 with cysteine-rich peptides, Org. Lett. 16 (2014) 3824-3827, http://dx.doi.org/10.1021/ ol501721j.

[12] S. Irtegun, R. Wood, K. Lackovic, J. Schweiggert, Y.M. Ramdzan, D.S.C. Huang, T.D. Mulhern, D.M. Hatters, A biosensor of SRC family kinase conformation by exposable tetracysteine useful for cell-based screening, ACS Chem. Biol. 9 (2014) 1426-1431, http://dx.doi.org/10.1021/cb500242q.

[13] G. Ponterini, A. Martello, G. Pavesi, A. Lauriola, R. Luciani, M. Santucci, M. Pelà G. Gozzi, S. Pacifico, R. Guerrini, G. Marverti, M.P. Costi, D. D’Arca, Intracellular quantitative detection of human thymidylate synthase engagement with an unconventional inhibitor using tetracysteine-diarsenical-probe technology, Sci. Rep. 6 (2016) 27198, http://dx.doi.org/10.1038/srep27198.

[14] A. Shevchenko, H. Tomas, J. Havli, J.V. Olsen, M. Mann, In-gel digestion for mass spectrometric characterization of proteins and proteomes, Nat. Protoc. 1 (2007) 2856-2860, http://dx.doi.org/10.1038/nprot.2006.468.

[15] W.J. Thompson, M.M. Appleman, Multiple cyclic nucleotide phosphodiesterase activities from rat brain, Biochemistry (Moscow) 10 (1971) 311-316

[16] Sybyl X 1.0 Tripos Inc, 1699 South Hanley Road, St Louis, Missouri 63144, USA

[17] K.Y. Zhang, G.L. Card, Y. Suzuki, D.R. Artis, D. Fong, S. Gillette, D. Hsieh, J Neiman, B.L. West, C. Zhang, M.V. Milburn, S.H. Kim, J. Schlessinger, G.A Bollag, Glutamine switch mechanism for nucleotide selectivity by phosphodiesterases, Mol. Cell 15 (July (2)) (2004) 279-286.

[18] MOE, Chemical Computing Group Inc., Montreal H3A 2R7, Canada. http:// www.chemcomp.com.

[19] A. Prandi, S. Franchini, L.I. Manasieva, P. Fossa, E. Cichero, G. Marucci, M. Buccioni, A. Cilia, L. Pirona, L. Brasili, Synthesis, biological evaluation, and docking studies of tetrahydrofuran- cyclopentanone- and cyclopentanol-based ligands acting at adrenergic $\alpha_{1}$ and serotonine 5-HT1A receptors, J. Med. Chem. 55 (2012) 23-36, http://dx.doi.org/10.1021/ jm200421e.

[20] S. Franchini, U.M. Battisti, A. Prandi, A. Tait, C. Borsari, E. Cichero, P. Fossa, A. Cilia, O. Prezzavento, S. Ronsisvalle, G. Aricò, C. Parenti, L. Brasili, Scouting new sigma receptor ligands: synthesis, pharmacological evaluation and molecular modeling of 1, 3-dioxolane-based structures and derivatives, Eur. J. Med. Chem. 112 (2016) 1-19, http://dx.doi.org/10.1016/j.ejmech.2016.01.059.

[21] Z.X. Wang, An exact mathematical expression for describing competitive binding of two different ligands to a protein molecule, FEBS Lett. 360 (1995) 111-114.

[22] K.S.S. Lee, C. Morisseau, J. Yang, P. Wang, S.H. Hwang, B.D. Hammock, Förster resonance energy transfer competitive displacement assay for human soluble epoxide hydrolase, Anal. Biochem. 434 (2013) 259-268, http://dx.doi.org/10. 1016/j.ab.2012.11.015.

[23] J.D. Corbin, S.H. Francis, Pharmacology of phosphodiesterase-5 inhibitors, Int. J. Clin. Pract. 56 (2002) 453-459.

[24] B.J. Sung, K.Y. Hwang, Y.H. Jeon, J.I. Lee, Y.S. Heo, J.H. Kim, J. Moon, J.M. Yoon, Y.L. Hyun, E. Kim, S.J. Eum, S.Y. Park, J.O. Lee, T.G. Lee, S. Ro, J.M. Cho, Structure of the catalytic domain of human phosphodiesterase 5 with bound drug molecules, Nature 425 (2003) 98-102, http://dx.doi.org/10.1038/ nature01914.

[25] T.L. Fink, S.H. Francis, A. Beasley, K.A. Grimes, J.D. Corbin, Expression of an active, monomeric catalytic domain of the cGMP-binding cGMP-specific phosphodiesterase (PDE5), J. Biol. Chem. 274 (1999) 34613-34620.

[26] C.C. Spagnuolo, R.J. Vermeij, E.A. Jares-Erijman, Improved photostable FRET-competent biarsenical-tetracysteine probes based on fluorinated fluoresceins, J. Am. Chem. Soc. 128 (2006) 12040-12041, http://dx.doi.org/10 $1021 / \mathrm{ja063212q}$.

[27] G.L. Card, B.P. England, Y. Suzuki, D. Fong, B. Powell, B. Lee, C. Luu, M. Tabrizizad, S. Gillette, P.N. Ibrahim, D.R. Artis, G. Bollag, M.V. Milburn, S.H. Kim, J. Schlessinger, K.Y.J. Zhang, Structural basis for the activity of drugs that inhibit phosphodiesterases, Struct. Lond. Engl. 1993 (12) (2004) 2233-2247, http://dx.doi.org/10.1016/j.str.2004.10.004.

[28] P. Wu, L. Brand, Resonance energy transfer: methods and applications, Anal. Biochem. 218 (1994) 1-13.

[29] J.R. Lakowicz, Principles of Fluorescence Spectroscopy, 3rd ed., Springer, New York, 2006.

[30] M.A. Blount, A. Beasley, R. Zoraghi, K.R. Sekhar, E.P. Bessay, S.H. Francis, J.D. Corbin, Binding of tritiated sildenafil, tadalafil, or vardenafil to the phosphodiesterase-5 catalytic site displays potency, specificity, heterogeneity, and cGMP stimulation, Mol. Pharmacol. 66 (2004) 144-152, http://dx.doi.org/10.1124/mol.66.1.144. 